\title{
Sequential development of flagellar defects in spermatids and epididymal spermatozoa of selenium-deficient rats
}

\author{
Gary E Olson ${ }^{1}$, Virginia P Winfrey ${ }^{1}$, Kristina E Hill ${ }^{2}$ and Raymond F Burk ${ }^{2}$ \\ ${ }^{1}$ Department of Cell and Developmental Biology and ${ }^{2}$ Division of Gastroenterology, Department of Medicine, \\ Vanderbilt University, Nashville, Tennessee 37232, USA \\ Correspondence should be addressed to Gary E Olson; Email: gary.olson@vanderbilt.edu
}

\begin{abstract}
In this study cauda epididymal spermatozoa of rats maintained on a selenium-deficient diet for $\mathbf{5}$ and 7 months exhibited an array of flagellar defects. Spermatids and spermatozoa were analyzed by light and electron microscopy to define the appearance of flagellar abnormalities during spermiogenesis and post-testicular sperm development. Late spermatids of seleniumdeficient rats displayed normal structural organization of the flagellar plasma membrane, axoneme, outer dense fibers, fibrous sheath and annulus, but they exhibited a premature termination of the mitochondrial sheath. A comparison of late spermatids and caput epididymal spermatozoa revealed that a late step in flagellar differentiation was the structural remodeling of the annulus and its accompanying fusion with both the fibrous sheath and the mitochondrial sheath. In selenium-deficient animals, however, the annulus failed to fuse with the mitochondrial sheath, generating an apparent weak point in the flagellum. After epididymal passage, cauda epididymal spermatozoa of selenium-deficient animals also exhibited extensive flagellar disorganization resulting from the apparent sliding and extrusion of specific outer dense fiber-doublet microtubule complexes from the proximal and the distal ends of the mitochondrial sheath and the accompanying loss of the midpiece plasma membrane. Only fiber complex number 4 was extruded proximally, whereas fibers 4, 5, 6 and 7 were extruded from the mitochondrial sheath-deficient posterior midpiece. Axonemal fibers 8, 9, 1, 2 and 3 retained their normal geometric relationships. These data suggest that the known loss of male fertility in selenium deficiency results from the sequential development of sperm defects expressed during both spermiogenesis and maturation in the epididymis.

Reproduction (2004) 127 335-342
\end{abstract}

\section{Introduction}

Selenium (Se) is an essential dietary micronutrient required for maintenance of male fertility (Maiorino et al. 1999). Early light microscopic studies established that epididymal spermatozoa of selenium-deficient animals exhibit a reduction or loss of motility and display flagellar defects localized primarily to the midpiece. The abnormalities include both hairpin-like bends of the flagellum as well as breaks and/or separation of its axial fibers (McCoy \& Weswig 1969, Wu et al. 1973, 1979, Wallace et al. 1983a, Watanabe \& Endo 1991). Selenium deficiency also reduces testis size, and prolonged deficiency results in atrophy of the seminiferous epithelium (Sprinker et al. 1971, Wallace et al. 1983a, Behne et al. 1996, MarinGuzman et al. 2000). In rats, ${ }^{75} \mathrm{Se}$ administered as sodium selenite by intraperitoneal injection is taken up by the testis and becomes concentrated in the sperm midpiece (Brown \& Burk 1973), consistent with a role in flagellar function. Biochemical analysis of fractionated spermatozoa demonstrated that the bulk of the ${ }^{75} \mathrm{Se}$ is incorporated in a flagellar polypeptide of $M_{\mathrm{r}} \approx 17000-$ 20000 (Calvin 1978), which, in the rat, is maximally expressed in step 7 through step 12 spermatids (Calvin et al. 1987). Subsequent analyses localized the selenoprotein to the midpiece, where it represents a major polypeptide of the mitochondrial capsule (Calvin \& Cooper 1979, Pallini \& Baccetti 1979, Pallini et al. 1979, Calvin et al. $1981 a, b)$, a rigid structural complex (Hrudka 1978), which becomes stabilized by disulfide bonds formed as spermatozoa undergo post-testicular maturation in the epididymis (Bedford \& Calvin 1974). Two candidate structural proteins of the sperm mitochondrial capsule have been identified. The first, termed 'sperm mitochondrial capsule protein $(\mathrm{SMCP})$ ', is a cysteine-rich protein of $M_{\mathrm{r}} \approx 22000$, but its deduced sequence contains no selenocysteine (Adham et al. 1996, Cataldo et al. 1996, Herr et al. 1999). The second is phospholipid hydroperoxide glutathione peroxidase (PHGPx; also termed 'GSHPx-4'), a selenocysteine-containing selenoprotein of $M_{\mathrm{r}} \approx 22000$ (Godeas 
et al. 1997, Ursini et al. 1999). PHGPx has been proposed to play a dual role in spermatozoa, primarily as an active enzyme protecting spermatozoa against lipid peroxidation and secondarily as an enzymatically inactive protein that performs a structural role in forming the mitochondrial capsule (Ursini et al. 1999).

Ultrastructural analyses of spermatozoa of seleniumdeficient animals have identified structural irregularities in the mitochondrial sheath, including abnormally shaped mitochondria, as well as supernumerary or deficient elements of the flagellar $9+9+2$ axoneme-outer dense fiber complex (Wu et al. 1979, Wallace et al. 1983a,b). Although the midpiece mitochondria of cauda epididymal spermatozoa from selenium-deficient mice apparently possess an insoluble capsule (Wallace et al. 1983a), it appears smaller and more fragile than those of control animals (Wallace et al. 1983b). Some infertile human males exhibit reduced sperm PHGPx levels, and ultrastructural defects in their midpiece mitochondria have been reported, supporting the role of selenoproteins in sperm function (Imai et al. 2001, Foresta et al. 2002). A preliminary light microscopic analysis of spermatozoa of selenium-deficient mice suggested that the flagellar bends detected in cauda spermatozoa occur less frequently in caput spermatozoa and are not evident in testicular spermatozoa (Wallace et al. 1983a). However, the mechanisms underlying the development of multiple flagellar defects in spermatozoa of selenium-deficient animals are poorly understood. To identify the earliest flagellar lesion(s) in selenium-deficient animals and the sequential development of secondary flagellar defects, we utilized light and electron microscopy to examine spermatids and epididymal spermatozoa of selenium-deficient and control rats. The data provide new insights into key steps of flagellar differentiation, in both normal and selenium-deficient animals, that are necessary to maintain intact flagellar architecture in mature epididymal spermatozoa.

\section{Materials and Methods}

\section{Animals}

Care and use of animals conformed to National Institutes of Health $(\mathrm{NIH})$ guidelines for humane animal care and use in research, and all protocols were approved by the institutional Animal Care and Use Committee. Animals were housed in a central animal care facility (approved by the Association for Assessment and Accreditation of Laboratory Animal Care) supervised by university veterinarians. Weanling male Sprague-Dawley rats (Harlan Sprague Dawley, Indianapolis, IN, USA) were fed ad libitum a torula yeast-based selenium-deficient diet or the same diet supplemented with $0.25 \mathrm{mg}$ selenium $/ \mathrm{kg}$ body weight as sodium selenate (Burk 1987). They were housed in a room with a 12-h:12-h light-dark cycle, and tap water was provided ad libitum. Animals were maintained for either 5 or 7 months on the selenium-deficient or control diets and then killed using sodium pentobarbital anesthesia $(65 \mathrm{mg} / \mathrm{kg}$ body weight administered intraperitoneally) and exsanguination by aortic puncture. Four control and four selenium-deficient rats, which were active and in apparent overall good health, were examined for each time point.

\section{Glutathione peroxidase (GSHPx) assays}

Blood and liver samples were prepared for determination of GSHPx activity, as their levels indicate overall selenium status. Blood was treated with disodium EDTA $(1 \mathrm{mg} / \mathrm{ml})$ to prevent coagulation, centrifuged and the plasma saved. Liver samples were homogenized in nine volumes of

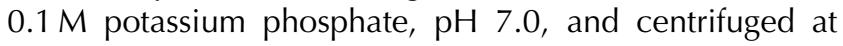
$13000 \mathrm{~g}$, and the supernatant fluid was saved. Glutathione peroxidase activity of blood plasma and the liver cytosol were measured using $0.25 \mathrm{mM}$ hydrogen peroxide as substrate (Lawrence \& Burk 1976).

\section{Light and electron microscopy}

The testis and the caput and cauda epididymides were prepared for both light and electron microscopy. For light microscopic analysis, tissues were minced in PBS or Hepes-buffered saline (HBS) (PBS $=145 \mathrm{mM} \mathrm{NaCl}$, $10 \mathrm{mM}$ sodium phosphate, $\mathrm{pH} 7.4 ; \mathrm{HBS}=145 \mathrm{mM} \mathrm{NaCl}$, $5 \mathrm{mM}$ Hepes, $\mathrm{pH}$ 7.4) to obtain a cell suspension. Aliquots of the suspension were examined by phase-contrast microscopy to assess sperm motility, and the remainder was fixed at $4{ }^{\circ} \mathrm{C}$ by the addition of two volumes of $4 \%$ formaldehyde, $0.1 \mathrm{M}$ sodium phosphate buffer, $\mathrm{pH}$ 7.4. At least 100 cells per sample were examined with a Zeiss Axiophot (Zeiss Instruments, Thornwood, NY), and images were recorded using a Spot 2 digital camera (Diagnostic Instruments Inc., Sterling Heights, MI, USA).

Tissues for electron microscopy were fixed at $4{ }^{\circ} \mathrm{C}$ with $4 \%$ glutaraldehyde in $0.1 \mathrm{M}$ sodium cacodylate buffer, $\mathrm{pH}$ 7.4, postfixed with $1 \% \mathrm{OsO}_{4}$ in cacodylate buffer, dehydrated in an ethanol series, equilibrated in propylene oxide and then embedded in epoxy resin. Thin sections were stained with uranyl acetate and lead citrate and examined in an Hitachi $\mathrm{H}-800$ electron microscope.

\section{Results}

\section{Reduced plasma and liver GSHPx in selenium-} deficient animals

Plasma and liver glutathione peroxidase activity of rats maintained on a selenium-deficient diet for 5 or 7 months was less than $1 \%$ of control rats (Table 1 ). This depression of GSHPx activity is evidence of severe selenium deficiency (Burk 1987).

\section{Flagellar abnormalities in cauda epididymal spermatozoa of selenium-deficient animals}

Cauda epididymal spermatozoa of control animals, fed a selenium-supplemented torula yeast diet for either 
Table 1 Glutathione peroxidase activity of rat plasma and liver.

\begin{tabular}{lcc}
\hline Diet & Plasma GSHPx & Liver GSHPx \\
\hline Selenium-deficient & $4 \pm 2$ & $4 \pm 1$ \\
Control & $2140 \pm 230$ & $400 \pm 100$ \\
\hline
\end{tabular}

Values are means \pm S.D. for four rats maintained on selenium-deficient diet for seven months. Activity units are nmol NADPH oxidized per min per ml plasma or per mg protein.

5 or 7 months, appeared structurally normal by phasecontrast microscopy (Fig. 1a), whereas spermatozoa from both the 5- and 7-month selenium-deficient rats displayed similar flagellar defects (Fig. 1b). All spermatozoa of both 5- and 7-month selenium-deficient animals exhibited an abrupt narrowing of the posterior midpiece that resulted from the premature termination of the mitochondrial sheath; gaps in the more proximal regions of the mitochondrial sheath were only occasionally observed. A second and frequent abnormality related to the missing mitochondrial sheath segment was a separation and/or extrusion of the underlying fibers of the axoneme-outer dense fiber complex. The projection of the distal end of some fibers from the mitochondrial sheath-deficient midpiece suggested their active extrusion from the principal piece. Other defects evident in some, but not all, spermatozoa, included an extrusion of flagellar fiber(s) from the neck region and the presence of kinks at the head-tail junction (Fig. 1b).

Cauda epididymal spermatozoa from seleniumdeficient animals exhibited specific ultrastructural defects in both the flagellar principal piece and midpiece. In the principal piece the fibrous sheath appeared normal and the plasma membrane was typically present. However, many flagellar profiles lacked a full complement of nine axonemal doublet microtubules and their associated outer dense fibers (Fig. 2a). Doublet microtubule-outer dense fiber complexes 8, 9, 1, 2 and 3, as well as the central pair microtubules, remained within the fibrous sheath lumen (Fig. 2a) and retained their normal geometric relationships. In contrast, various combinations of doublet microtubule-outer dense fiber complex numbers 4-7 were absent; presumably, they represent the extruded fibers seen by phase-contrast microscopy (Fig. 2a). Extruded axonemal doublet microtubules remained attached to their companion outer dense fiber (Fig. 2a and b). Unlike the principal piece, the midpiece frequently lacked an intact plasma membrane (Fig. 2b). In the proximal midpiece the most typical defect was the specific absence of doublet microtubule-outer dense fiber complex number 4 (Fig. 2b). Occasional profiles of the proximal midpiece exhibited variable numbers of doublet microtubule-outer dense fiber complexes interposed between the mitochondrial sheath and plasma membrane (Fig. 2c). These represented multiple profiles of doublet microtubule-outer dense fiber complexes 47, which assumed a coiled configuration following extrusion from the principal piece. Compared with the proximal midpiece, a more frequent absence and disorganization of flagellar fibers was evident in the distal midpiece segment that lacked the mitochondrial sheath (Fig. 2c). The junction of the midpiece and principal piece is characterized by a ring-like annulus (Fawcett 1975), a filamentous structure that is adherent to the posterior margin of the mitochondrial sheath, to the anterior margin of the fibrous sheath and to the overlying plasma membrane. In spermatozoa of selenium-deficient animals, the annulus occupied its normal position at the midpiece-principal piece junction and was adherent to the proximal end of the fibrous sheath, but not to the mitochondrial sheath (Fig. 2d).
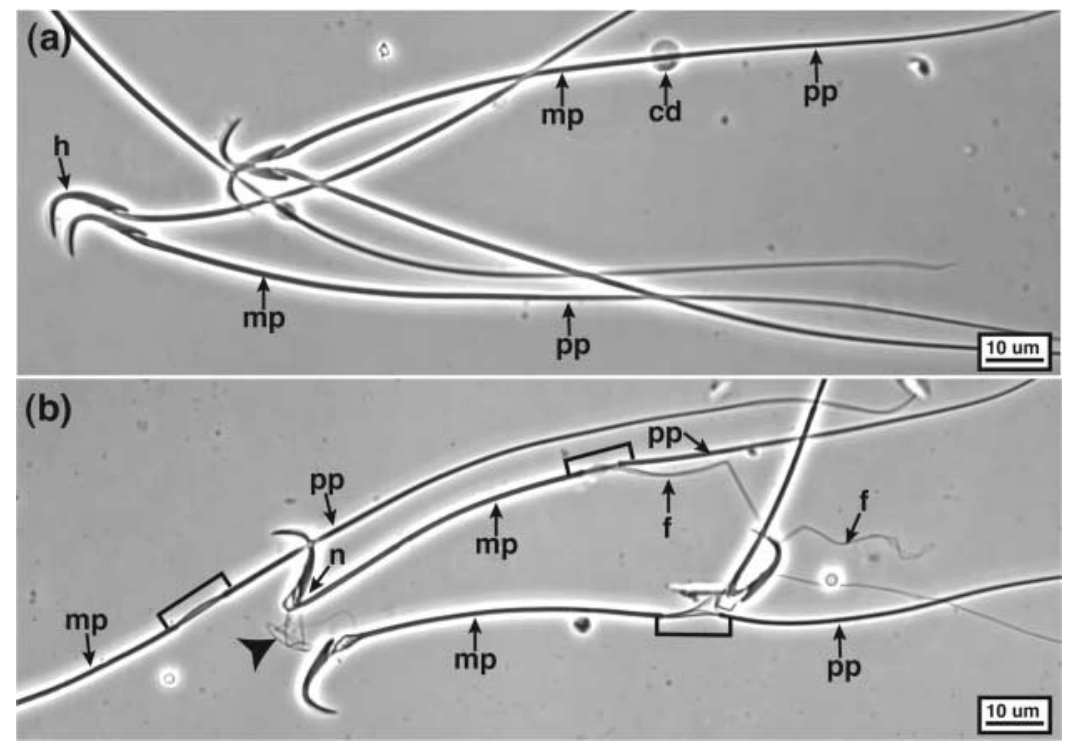

www.reproduction-online.org
Figure 1 (a) Phase-contrast photomicrograph showing intact flagella of cauda epididymal spermatozoa from control rat. $\mathrm{h}=$ sperm head; $\mathrm{mp}=$ midpiece; $\mathrm{cd}=$ cytoplasmic droplet; and $\mathrm{pp}=$ principal piece. Bar $=10 \mu \mathrm{m}$. (b) Phase-contrast photomicrograph of cauda epididymal spermatozoa from seleniumdeficient rat. All spermatozoa exhibit a narrowing of the posterior midpiece (bracket), reflecting the absence of the mitochondrial sheath. Note the separation of the flagellar fibers ( $f$ ) and their protrusion from the mitochondrial sheath-deficient midpiece segment. One spermatozoon shows a fiber extruded from the neck (n) region (arrows). Bar $=10 \mu \mathrm{m}$. 


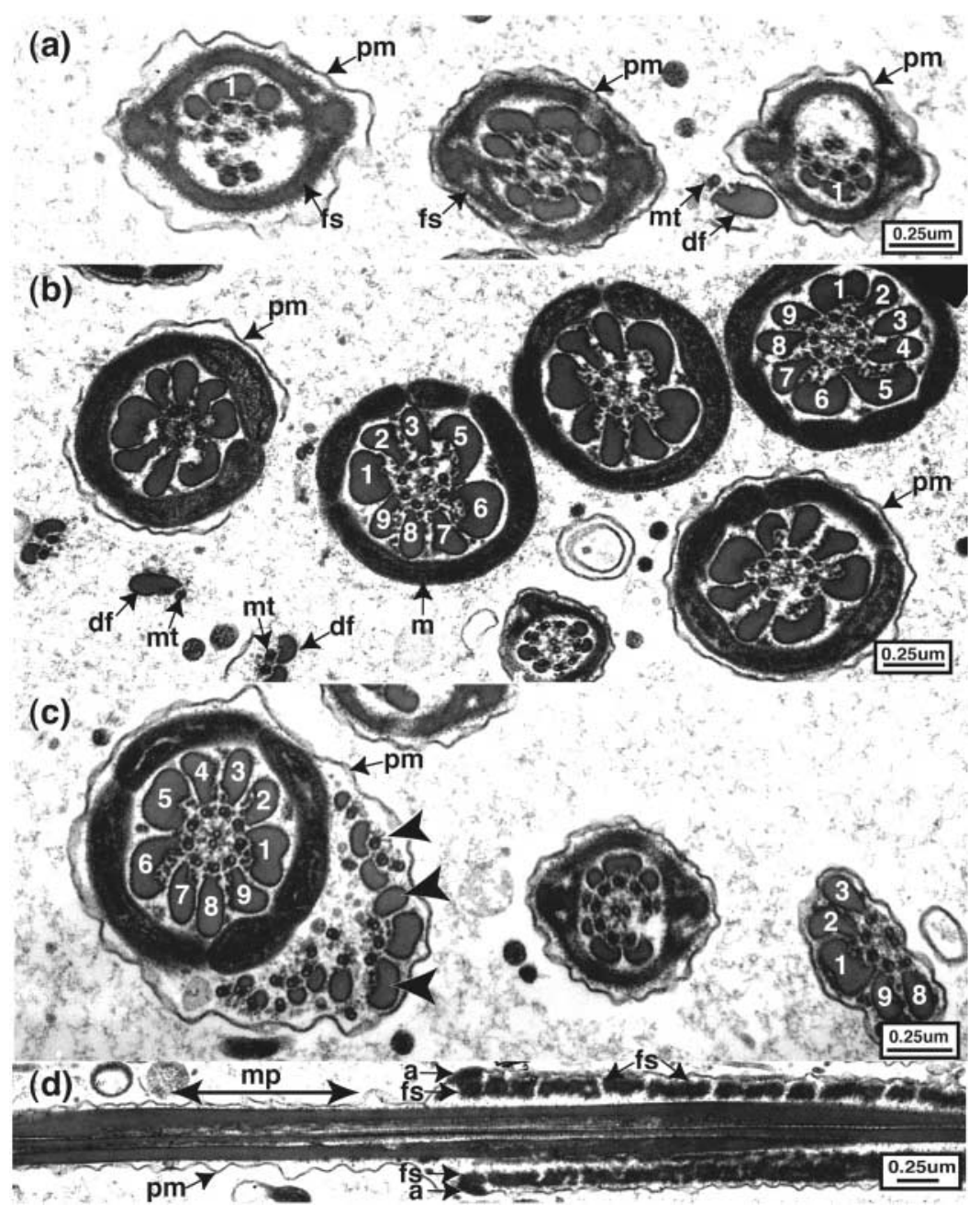

Figure 2 (a) Electron micrograph showing principal piece cross-sections of cauda epididymal spermatozoa from a selenium-deficient rat. The plasma membrane $(\mathrm{pm})$ and fibrous sheath $(\mathrm{fs})$ appear intact in each principal piece. One flagellum (center) shows a normal $9+2$ axoneme and complement of outer dense fibers, whereas the principal piece on the left lacks axonemal doublet microtubule number 4 and the one at the far right lacks doublet microtubules $4-7$. Note that the extruded doublet microtubules $(\mathrm{mt})$ remain adherent to the companion outer dense fibers (df). Bar $=0.25 \mu \mathrm{m}$. (b) Electron micrograph showing cross-sections of the midpiece of several cauda epididymal spermatozoa of a selenium-deficient rat. The plasma membrane (pm) appears intact on one spermatozoon, but is either disrupted or absent in the others. One midpiece possesses an intact axoneme-outer dense fiber complex (upper right), whereas all other midpieces lack doublet microtubule-outer dense fiber complex number 4. Extruded doublet microtubule (mt)-outer dense fiber (df) complexes remain linked together. $\mathrm{m}=$ mitochondrial sheath. Bar $=0.25 \mu \mathrm{m}$. (c) Cauda epididymal spermatozoa of selenium-deficient rat showing a profile of the distal midpiece lacking the mitochondrial sheath, and containing only doublet microtubule-outer dense fiber complex numbers 8, 9, 1, 2 and 3 (lower right). A second midpiece profile shows an accumulation of doublet microtubule-outer dense fiber complexes (arrowheads) between the plasma membrane (pm) and mitochondrial sheath. Note that the principal piece profile lacks doublet microtubule number 4. Bar $=0.25 \mu \mathrm{m}$. (d) Longitudinal section showing midpiece-principal piece junction of cauda epididymal spermatozoon from a selenium-deficient rat. The mitochondrial sheath is absent from the terminal midpiece (mp) segment. The annulus (a) is adherent to the plasma membrane $(\mathrm{pm})$ and the proximal margin of the fibrous sheath (fs). Bar $=0.25 \mu \mathrm{m}$.

\section{Development of flagellar abnormalities in selenium- deficiency during spermiogenesis and epididymal maturation}

Spermatids and caput epididymal spermatozoa were examined to define the temporal development of the selenium-dependent flagellar defects identified in cauda epididymal spermatozoa. Late spermatids and testicular spermatozoa of selenium-deficient rats displayed a gap in the posterior midpiece, reflecting the absence of the mitochondrial sheath (Fig. 3a); however, there was no separation or extrusion of the underlying axonemal doublet microtubule-outer dense fiber complexes from either the gap region or the neck region. They also displayed an intact plasma membrane, axoneme and complement of outer dense fibers, and the $9+9+2$ geometry of the axoneme-outer dense fiber complex was maintained even in the distal midpiece lacking the mitochondrial sheath (Fig. 3b). In late spermatids of both selenium-deficient and control rats, the annulus exhibited a semicircular crosssectional profile and a bipartite appearance, with the dense annular component attached to the plasma membrane, while the less dense component faced anteriorly toward the mitochondrial sheath (Fig. 3c and d). An invagination of the plasma membrane, the retroannular recess (Fawcett et al. 1970), separated the annulus and fibrous sheath by a similar distance in spermatids of both control and selenium-deficient animals. Likewise, the annulus was separated from the mitochondrial sheath in both seleniumdeficient and control spermatids (Fig. 3c and d). However, the separation was up to several micrometers in seleniumdeficient spermatids due to the premature termination of the mitochondrial sheath (Fig. 3c), whereas in controls it represented only a short gap (Fig. 3d).

Caput epididymal spermatozoa from selenium-deficient animals exhibited a variable length gap in the posterior mitochondrial sheath but rarely a separation or extrusion of the underlying flagellar fibers (Fig. 4a). Although most caput spermatozoa from selenium-deficient rats possessed an intact midpiece plasma membrane and axonemeouter dense fiber complex, an impending initiation of flagellar disintegration was evidenced in some by extruded doublet microtubule-outer dense fiber complexes interposed between the plasma membrane and mitochondrial sheath (Fig. 4c). In comparison to 

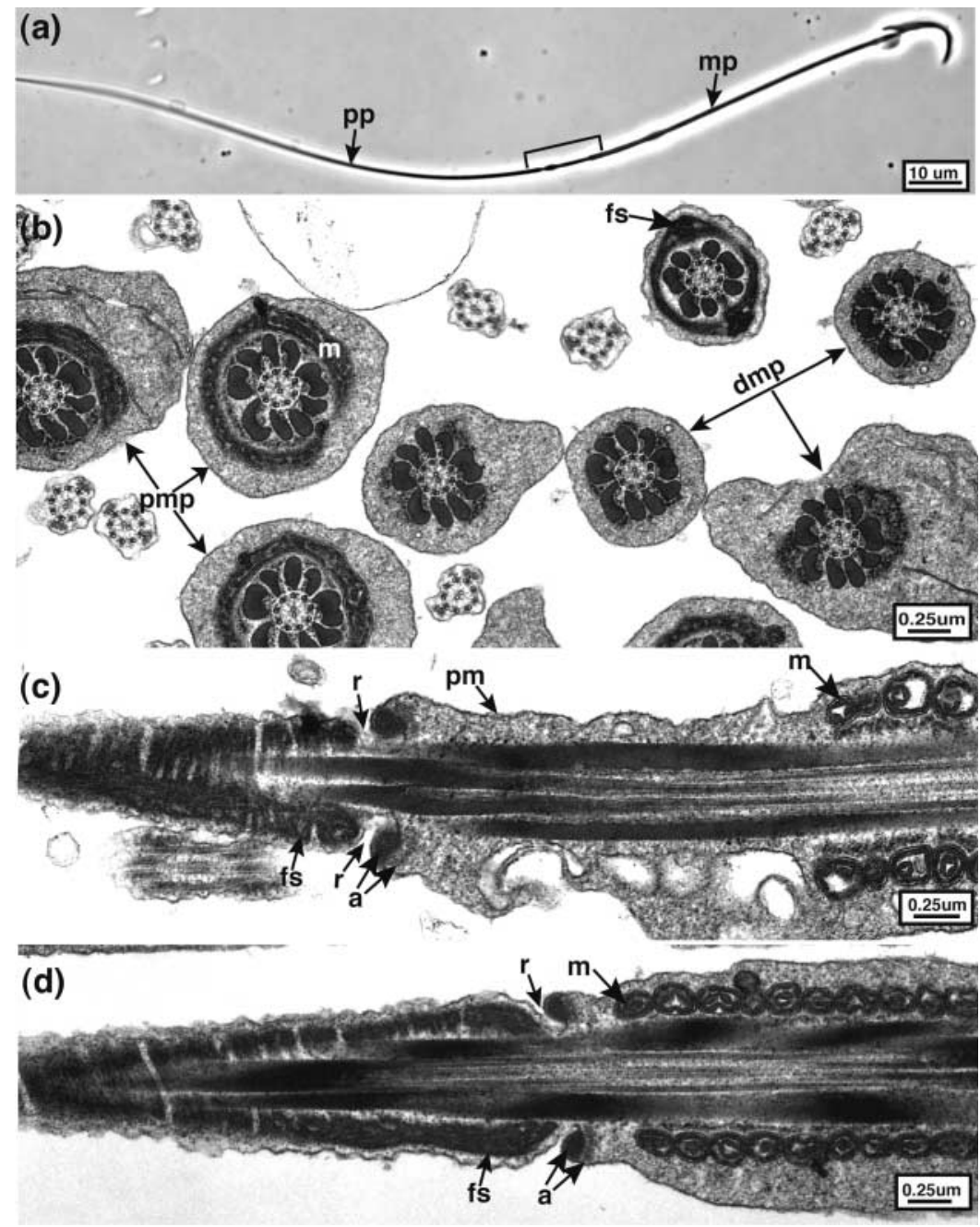

Figure 3 (a) Phase-contrast photomicrograph showing a late spermatid of a selenium-deficient rat. A gap is present in the posterior mitochondrial sheath (bracket). Bar $=10 \mu \mathrm{m}$. (b) Flagellar cross-sections of late spermatids from selenium-deficient animal. The axonemes and outer dense fibers appear intact in all flagellar segments. The proximal midpiece (pmp) possesses an intact mitochondrial sheath $(\mathrm{m})$, but it is absent in the distal midpiece (dmp). $\mathrm{fs}=$ fibrous sheath. Bar $=0.25 \mu \mathrm{m}$. (c) Longitudinal section of the midpiece-principal piece junction of a late spermatid from a selenium-deficient rat. The annulus (a) is bilayered in appearance (double arrows) and does not contact the truncated mitochondrial sheath $(m)$. An indentation of the plasma membrane, the retroannular recess (r), separates the annulus and fibrous sheath (fs). Bar $=0.25 \mu \mathrm{m}$. (d) Longitudinal section of the midpiece-principal piece junction of a late spermatid from a control rat. Note that the annulus (a) is separated from the fibrous sheath ( $\mathrm{fs}$ ) by the retroannular recess $(r)$ and that it is also separated from the adjacent mitochondrial sheath $(\mathrm{m})$. Bar $=0.25 \mu \mathrm{m}$. spermatids, the midpiece-principal piece junction of caput spermatozoa of selenium-deficient (Fig. 4b) and control (Fig. 4d) rats revealed a set of maturation-dependent structural changes in the annulus. The annulus exhibited a wedge-shaped, rather than semicircular, crosssectional profile and appeared homogeneous, rather than bipartite, in electron density. In addition the retroannular recess was absent, permitting the annulus to attach itself to the fibrous sheath. In control spermatozoa the annulus also had attached itself to the posterior margin of the mitochondrial sheath (Fig. 4d), whereas the annulus in selenium-deficient spermatozoa had not, so that the fibrous sheath, annulus and mitochondrial sheath failed to form a cohesive structural unit (Fig. 4b).

\section{Discussion}

This study demonstrates the temporal development of a set of flagellar defects during spermiogenesis and posttesticular sperm maturation in the selenium-deficient rat. The primary lesion involves a truncation of the spermatid mitochondrial sheath and its subsequent failure to fuse with the annulus, an event that normally welds the mitochondrial sheath and fibrous sheath into a continuous structural unit. This creates a structurally weak zone where microtubule-dense fiber complexes are extruded during flagellar disintegration in epididymal spermatozoa.

Selenium exerts its physiological activity as the amino acid selenocysteine, which is incorporated into the primary structure of selenoproteins (Stadtman 1996). The predominant sperm selenoprotein, PHGPx, is assembled into the mitochondrial capsule and is believed to have both enzymatic and structural functions (Ursini et al. 1999). This complex, which is unique to the spermatozoa of therian mammals, becomes extensively crosslinked by disulfide bonds formed as they mature in the epididymis (Bedford \& Calvin 1974). Although the functions of the mitochondrial capsule are unresolved, mitochondria of PHGPx-deficient human spermatozoa display reduced uptake of rhodamine 123, indicating altered membrane potential (Imai et al. 2001). Other sperm selenoproteins have not been well characterized. Spermatozoa do express thioredoxin (Miranda-Vizuete et al. 2001, Jimenez et al. 2002), an enzyme that 

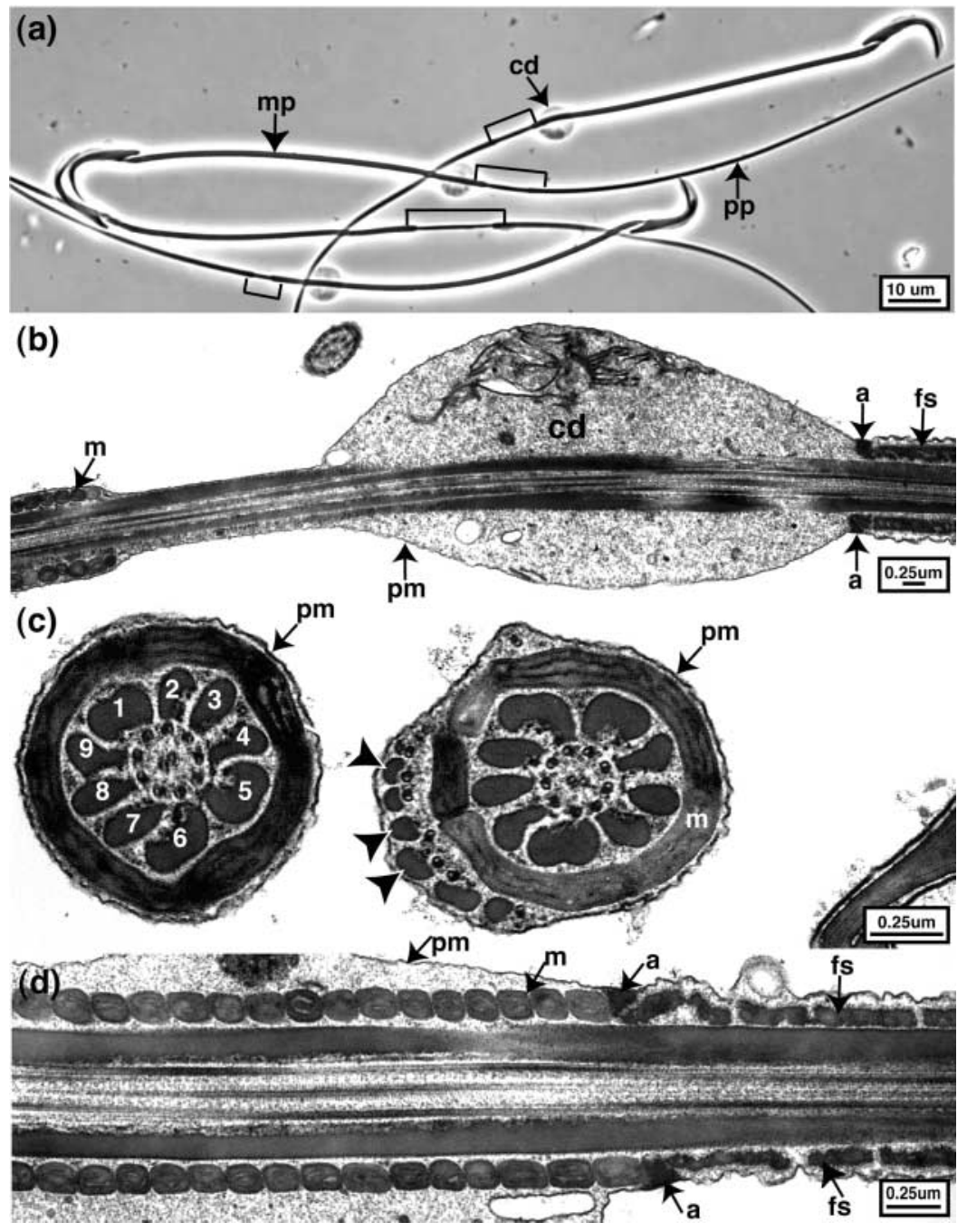

Figure 4 (a) Phase-contrast photomicrograph of caput epididymal spermatozoa from a selenium-deficient rat. Note the variable length of the mitochondrial sheath-deficient zone in the distal midpiece (brackets). $c d=$ cytoplasmic droplet; $\mathrm{mp}=$ midpiece; $\mathrm{pp}=$ principal piece. Bar $=10 \mu \mathrm{m}$. (b) Longitudinal section of the midpiece-principal piece junction of a caput epididymal spermatozoon of a selenium-deficient animal. Note the premature termination of the mitochondrial sheath $(\mathrm{m})$ and that the cytoplasmic droplet (cd) has migrated to the distal midpiece. Note also that the annulus (a) is attached to the fibrous sheath and that the retroannular recess is absent. Bar $=0.25 \mu \mathrm{m}$. (c) Cross-section of the midpiece of two caput epididymal spermatozoa from a selenium-deficient rat. One midpiece appears normal, but the second shows an accumulation of doublet microtubule-outer dense fiber complexes (arrowheads) between the plasma membrane $(\mathrm{pm})$ and mitochondrial sheath $(\mathrm{m})$. Bar $=0.25 \mu \mathrm{m}$. (d) Longitudinal section of the midpiece-principal piece junction of caput epididymal spermatozoon from a control rat. Note the wedge shape of the annulus (a) and the absence of the retroannular recess. The annulus attaches to both the fibrous sheath (fs) and mitochondrial sheath $(\mathrm{m})$. $\mathrm{pm}=$ plasma membrane. Bar $=0.25 \mu \mathrm{m}$. regulates protein disulfide status and whose activity requires thioredoxin reductase, a selenoprotein that is expressed in the testis (Sun et al. 1999) but remains to be demonstrated in spermatozoa. Thus, selenium deficiency may compromise the functional activity of the sperm glutathione peroxidase and/or the thioredoxin systems, resulting in oxidative damage and altered disulfide status, and may also affect the mitochondrial remodeling that occurs during mitochondrial sheath formation (André, 1962, DeMartino et al. 1979).

It remains uncertain why the secondary defects in flagellar structure are typically detected only after spermatozoa leave the caput epididymidis. Flagellar disintegration involves the proximal sliding of axonemal doublet microtubule-outer dense fiber complex numbers 4-7, and their extrusion from either the proximal end or the distal, mitochondrial sheath-deficient end of the midpiece. Doublet microtubule-outer dense fiber complex number 4 appears to initiate flagellar disintegration, as it was the first one extruded from the principal piece and the only one occasionally extruded from the proximal midpiece. The key flagellar defect for doublet microtubule-outer dense fiber extrusion appears to be the missing segment of the mitochondrial sheath. An identical pattern of flagellar disintegration and proximal sliding of fibers 4-7 from the fibrous sheath has been demonstrated in vitro for cauda epididymal rat spermatozoa following removal of the midpiece mitochondrial sheath with Triton X-100 and dithiothreitol and reactivation of flagellar motility with ATP (Olson \& Linck 1977). Links between fibers 8 and 3 and the continuous columns of the fibrous sheath (Fawcett 1975) prevent their sliding from it, but it is not clear why fibers 9,1 and 2 are not extruded and retain their normal structural relationships within the principal piece.

Flagellar disintegration coincides with the development of the potential for more vigorous motility in mature epididymal spermatozoa (Bedford 1975, Eddy \& O'Brien 1993). However, accumulated oxidative damage either from dysfunction of the sperm antioxidant systems or from loss of the protective function of the cauda environment (Bedford 1979) may be a key factor. Principal cells of the cauda, as compared with the caput, epididymidis show higher expression of the selenoprotein, glutathione peroxidase type 3 (Schwaab et al. 1998a,b), and 
selenium-deficiency may affect GSHPx-3 levels, resulting in oxidative damage to the cauda. Thus, the spectrum of sperm defects seen in selenium-deficient rats may reflect dysfunction of both spermiogenesis and the cauda epididymidis.

Understanding the molecular basis for the flagellar defects in the selenium-deficient animals may provide insights into the mechanism(s) of some cases of male infertility. Missing axonemal microtubules and outer dense fibers represent a common defect noted in a variety of pathologic conditions in human and animal spermatozoa (Zamboni 1987, Ryder et al. 1990, Chemes 2000). Examination of their micrographs indicates that various combinations of doublet microtubule-outer dense fibers 4-7 are typically missing in the principal piece and sometimes the midpiece, and this reflects the specific pattern of axonemal disintegration described here. In several genetic defects, such as the bovine Dag defect (Blom \& BirchAndersen 1966, Koefoed-Johnsen \& Pedersen 1971), the wobbler mouse (Leestma \& Sepsenwol 1980) and the t-allele mouse (Olds 1971, Dooher \& Bennett 1977), or in rats treated with gossypol (Oko \& Hrudka 1982) and rhesus monkeys treated with 1-(2,4-dichlorobenzyl)-indazole-3-carboxylic acid (Lobl \& Mathews 1978), sperm axonemal morphology appears normal in the testis and/or proximal epididymis, but spermatozoa in the distal cauda epididymidis exhibit a similar pattern of axonemal disintegration. Interestingly, both a mitochondrial sheath truncation and an assortment of mitochondrial defects have been reported in spermatids and epididymal spermatozoa of gossypol-treated rats (Nadakavukaren et al. 1979, Hadley et al. 1981, Oko \& Hrudka 1982, Hoffer 1983, Tanphaichitr et al. 1984, Chenoweth et al. 2000). Thus, a number of reported sperm flagellar abnormalities that appear in the epididymis may have a common underlying mechanistic origin, and it will be interesting to determine whether the altered expression or function of spermatozoan and/or epididymal selenoproteins plays a central role in the development of these sperm defects.

\section{Acknowledgement}

This study was supported by NIH grants HD44863 and HD20419 (G.E.O.) and ES02497 (R.F.B.).

\section{References}

Adham IM, Tessmann D, Soliman KA, Murphy D, Kremling H, Szpirer C \& Engel W 1996 Cloning, expression, and chromosomal localization of the rat mitochondrial capsule selenoprotein gene (MCS): the reading frame does not contain potential UGA selenocysteine codons. DNA and Cell Biology 15 159-166.

André J 1962 Contribution á la connaissance du chondriome. Étude de ses modifications ultrastructurales pendant la spermatogenèse. Journal of Ultrastructure Research. Supplement 3 1-185.

Bedford JM 1975 Maturation, transport, and fate of spermatozoa in the epididymis. In Handbook of Physiology: Endocrinology, Male Reproductive System, pp 303-317. Eds RO Greep \& EB Astwood. Washington, DC: Waverly Press.
Bedford JM 1979 Evolution of the sperm maturation and sperm storage functions of the epididymis. In The Spermatozoon, pp 7-22. Eds DW Fawcett \& JM Bedford. Baltimore, MD: Urban \& Schwarzenberg.

Bedford JM \& Calvin HI 1974 Changes in SS-linked structures of the sperm tail during epididymal maturation, with comparative observations in sub-mammalian species. Journal of Experimental Zoology 187 181-204.

Behne D, Weiler H \& Kyriakopoulos A 1996 Effects of selenium deficiency on testicular morphology and function in rats. Journal of Reproduction and Fertility 106 291-297.

Blom E \& Birch-Andersen A 1966 The ultrastructure of a new hereditary sterilizing defect (the 'Dag defect') in the bull sperm tail. Excerpta Medica International Congress Series 133 602-605.

Brown DG \& Burk RF 1973 Selenium retention in tissues and sperm of rats fed a torula yeast diet. Journal of Nutrition 102 102-108.

Burk RF 1987 Production of selenium deficiency in the rat. Methods in Enzymology 143 307-313.

Calvin HI 1978 Selective incorporation of selenium-75 into a polypeptide of the rat sperm tail. Journal of Experimental Zoology 204 $445-452$.

Calvin HI \& Cooper GW 1979 A specific selenopolypeptide associated with the outer membrane of rat sperm mitochondria. In The Spermatozoon, pp 135-140. Eds DW Fawcett \& JM Bedford. Baltimore, MD: Urban and Schwarzenberg.

Calvin HI, Cooper GW \& Wallace E 1981a Evidence that selenium in rat sperm is associated with a cysteine-rich structural protein of the mitochondrial capsules. Gamete Research 4 139-149.

Calvin HI, Wallace E \& Cooper GW $1981 b$ The role of selenium in the organization of the mitochondrial helix in rodent spermatozoa. In Selenium in Biology and Medicine, pp 319-324. Eds JE Spalholz, JL Martin \& HE Ganther. Westport, CT: AVI.

Calvin HI, Grosshans K, Musicant-Shikora SR \& Turner SI 1987 A developmental study of rat sperm and testis selenoproteins. Journal of Reproduction and Fertility 81 1-11.

Cataldo L, Baig K, Oko R, Mastrangelo MA \& Kleene KC 1996 Developmental expression, intracellular localization, and selenium content of the cysteine-rich protein associated with the mitochondrial capsules of mouse sperm. Molecular Reproduction and Development 45 320-331.

Chemes HE 2000 Phenotypes of sperm pathology: genetic and acquired forms in infertile men. Journal of Andrology 21 799-808.

Chenoweth PJ, Chase CC, Risco CA \& Larsen RE 2000 Characterization of gossypol-induced sperm abnormalities in bulls. Theriogenology 53 1193-1203.

DeMartino C, Floridi A, Marcante ML, Malorni W, ScorzaBarcellona P, Belloci M \& Silvestrini B 1979 Morphological, histochemical and biochemical studies on germ cell mitochondria of normal rats. Cell and Tissue Research 196 1-22.

Dooher GB \& Bennett D 1977 Spermiogenesis and spermatozoa in sterile mice carrying different lethal T/t locus haplotypes: a transmission and scanning electron microscopic study. Biology of Reproduction 17 269-288.

Eddy EM \& O'Brien DA 1993 The spermatozoon. In The Physiology of Reproduction, pp 29-77. Eds E Knobil \& JD Neill. New York: Raven Press.

Fawcett DW 1975 The mammalian spermatozoon. Developmental Biology 44 394-436.

Fawcett DW, Eddy EM \& Phillips DM 1970 Observations on the fine structure and relationships of the chromatoid body in mammalian spermatogenesis. Biology of Reproduction 2 129-153.

Foresta C, Flohe L, Garolla A, Roveri A, Ursini F \& Maiorino M 2002 Male fertility is linked to the selenoprotein phospholipid hydroperoxide glutathione peroxidase. Biology of Reproduction 67 967-971.

Godeas C, Tramer F, Micali F, Soranzo M, Sandri G \& Panfili E 1997 Distribution and possible novel role of phospholipid hydroperoxide glutathione peroxidase in rat epididymal spermatozoa. Biology of Reproduction 57 1502-1508. 
Hadley MA, Lin YC \& Dym M 1981 Effects of gossypol on the reproductive system of male rats. Journal of Andrology 2 190-199.

Herr JC, Thomas D, Bush LA, Coonrod S, Khole V, Howards SS \& Flickinger CJ 1999 Sperm mitochondria-associated cysteine-rich protein (SMCP) is an autoantigen in Lewis rats. Biology of Reproduction 61 428-435.

Hoffer AP 1983 Effects of gossypol on the seminiferous epithelium in the rat: a light and electron microscope study. Biology of Reproduction 28 1007-1020.

Hrudka F 1978 A morphological and cytochemical study on isolated sperm mitochondria. Journal of Ultrastructure Research $\mathbf{6 3}$ $1-19$.

Imai H, Suzuki K, Ishizaka K, Ichinose S, Oshima H, Okayasu I, Emoto K, Umeda M \& Nakagawa Y 2001 Failure of the expression of phospholipid hydroperoxide glutathione peroxidase in the spermatozoa of human infertile males. Biology of Reproduction 64 674-683.

Jimenez A, Oko R, Gustafsson JA, Spyrou G, Pelto-Huikko M \& Miranda-Vizuete A 2002 Cloning, expression and characterization of mouse spermatid specific thioredoxin-1 gene and protein. Molecular Human Reproduction 8 710-718.

Koefoed-Johnsen HH \& Pedersen H 1971 Further observations on the Dag-defect of the tail of the bull spermatozoon. Journal of Reproduction and Fertility 26 77-83.

Lawrence RA \& Burk RF 1976 Glutathione peroxidase activity in selenium-deficient rat liver. Biochemical and Biophysical Research Communications 71 952-958.

Leestma JE \& Sepsenwol S 1980 Sperm tail axoneme alterations in the Wobbler mouse. Journal of Reproduction and Fertility $\mathbf{5 8}$ $267-270$.

Lobl TJ \& Mathews J 1978 Effect of 1-(2,4-dichlorobenzyl)-indazole3 -carboxylic acid on sperm tails in rhesus monkeys. Journal of Reproduction and Fertility $\mathbf{5 2}$ 275-278.

Maiorino M, Flohe L, Roveri A, Steinert P, Wissing JB \& Ursini F 1999 Selenium and reproduction. Biofactors 10 251-256.

Marin-Guzman J, Mahan DC \& Pate JL 2000 Effect of dietary selenium and vitamin $\mathrm{E}$ on spermatogenic development in boars. Journal of Animal Science 78 1537-1543.

McCoy KEM \& Weswig PH 1969 Some selenium responses in the rat not related to vitamin E. Journal of Nutrition 98 383-389.

Miranda-Vizuete A, Ljung J, Damdimopoulos AE, Gustafsson J-A, Oko R, Pelto-Huikko M \& Spyrou G 2001 Characterization of Sptrx, a novel member of the thioredoxin family specifically expressed in human spermatozoa. Journal of Biological Chemistry 276 31567-31574.

Nadakavukaren MJ, Sorensen RH \& Tone JN 1979 Effect of gossypol on the ultrastructure of rat spermatozoa. Cell and Tissue Research $204293-296$.

Oko R \& Hrudka F 1982 Segmental aplasia of the mitochondrial sheath and sequelae induced by gossypol in rat spermatozoa. Biology of Reproduction 26 183-195.

Olds PJ 1971 Effect of the T locus on sperm ultrastructure in the house mouse. Journal of Anatomy 109 31-37.

Olson GE \& Linck RW 1977 Observations of the structural components of flagellar axonemes and central pair microtubules from rat sperm. Journal of Ultrastructure Research 61 21-43.

Pallini V \& Baccetti B 1979 Bull sperm selenium is bound to a structural protein of mitochondria. Journal of Submicroscopic Cytology 11 165-170.
Pallini V, Baccetti B \& Burrini AG 1979 A peculiar cysteinerich polypeptide related to some unusual properties of mammalian sperm mitochondria. In The Spermatozoon, pp 141-151. Eds DW Fawcett \& JM Bedford. Baltimore, MD: Urban and Schwarzenberg.

Ryder TA, Mobberley AA, Hughes L \& Hendry WF 1990 A survey of the ultrastructural defects associated with absent or impaired sperm motility. Fertility and Sterility 53 556-560.

Schwaab V, Faure J, Dufaure J-P \& Drevet JR 1998a GPx3: the plasma-type glutathione peroxidase is expressed under androgenic control in the mouse epididymis and vas deferens. Molecular Reproduction and Development $51362-372$.

Schwaab V, Lareyre JJ, Vernet P, Pons E, Faure J, Dufaure J-P \& Drevet JR 1998b Characterization, regulation of the expression and putative roles of two glutathione peroxidase proteins found in the mouse epididymis. Journal of Reproduction and Fertility Supplement 53 157-162.

Sprinker LH, Harr JR, Newberne PM, Whanger PD \& Weswig PH 1971 Selenium deficiency lesions in rats fed vitamin E supplemented rations. Nutrition Reports International 4 335-340.

Stadtman TC 1996 Selenocysteine. Annual Reviews in Biochemistry $6583-100$

Sun Q-A, Wu Y, Zappacosta F, Jeang K-T, Lee BJ, Hatfield DL \& Gladyshev VN 1999 Redox regulation of cell signaling by selenocysteine in mammalian thioredoxin reductases. Journal of Biological Chemistry $27424522-24530$.

Tanphaichitr N, Chen LB \& Bellve AR 1984 Direct effect of gossypol on TR-ST cells: perturbation of rhodamine 123 accumulation in mitochondria. Biology of Reproduction 31 1049-1060.

Ursini F, Heim S, Kiess M, Maiorino M, Roveri A, Wissing J \& Flohe L 1999 Dual function of the selenoprotein PHGPx during sperm maturation. Science 277 225-228.

Wallace E, Calvin HI \& Cooper GW 1983a Progressive defects observed in mouse sperm during the course of three generations of selenium deficiency. Gamete Research 4 377-387.

Wallace E, Cooper GW \& Calvin HI 1983b Effects of selenium deficiency on the shape and arrangement of rodent sperm mitochondria. Gamete Research 4 389-399.

Watanabe T \& Endo A 1991 Effects of selenium deficiency on sperm morphology and spermatocyte chromosomes in mice. Mutation Research 262 93-99.

Wu ASH, Oldfield JE, Shull LR \& Cheeke PR 1979 Specific effect of selenium deficiency on rat sperm. Biology of Reproduction $\mathbf{2 0}$ 793-798.

Wu SH, Oldfield JE, Whanger PD \& Weswig PH 1973 Effect of selenium, vitamin $\mathrm{E}$, and antioxidants on testicular function in rats. Biology of Reproduction 8 625-629.

Zamboni L 1987 The ultrastructural pathology of the spermatozoon as a cause of infertility: the role of electron microscopy in the evaluation of semen quality. Fertility and Sterility $\mathbf{4 8}$ 711-734.

Received 28 April 2003

First decision 1 July 2003

Revised manuscript received 6 October 2003

Accepted 26 November 2003 